\title{
Evidence that the gene encoding ZDHHC8 contributes to the risk of schizophrenia
}

\author{
Jun Mukai ${ }^{1,3}$, Hui Liu ${ }^{2,3}$, Rachel A Burt ${ }^{2}$, Dionne E Swor ${ }^{1}$, Wen-Sung Lai ${ }^{1,2}$, Maria Karayiorgou ${ }^{2}$ \& \\ Joseph A Gogos ${ }^{1}$
}

Using a relatively dense genetic map of 72 single-nucleotide polymorphisms (SNPs) distributed across the entire 1.5-Mb locus on chromosome 22q11 associated with susceptibilit to schizophrenia ${ }^{1,2}$, we previously identified two subregions that were consistently associated with the disease ${ }^{3,4}$. In the distal subregion, we detected an association signal with five neighboring SNPs distributed over a haplotypic block of 80 kb encompassing six known genes ${ }^{4}$. One of these five SNPs, rs175174, had the strongest association of all 72 SNPs that we tested. Here we show that rs 175174 regulates the level of the fully functional transcript by modulating the retention of intron 4 of the gene $Z D H H C 8$, which encodes a putative transmembrane palmitoyltransferase. Zdhhc8-knockout mice had a sexually dimorphic deficit in prepulse inhibition, a gene dosage-dependent decrease in exploratory activity in a new environment and a decreased sensitivity to the locomotor stimulatory effects of the psychomimetic drug dizocilpine (MK801). SNP rs175174 shows differences in transmission distortion between sexes in individuals with schizophrenia. Our results indicate that there is an unexpected connection between impaired palmitate modification of neuronal proteins and the psychiatric phenotypes associated with microdeletions of chromosome 22q11.

SNP rs175174 is located in intron 4 of $Z D H H C 8$ (previously annotated as KIAA1292; Fig. 1a,b), and the potential effect of rs175174 genotype on splicing warranted further investigation. We prepared total or poly $(\mathrm{A})^{+}$RNA from fresh (nontransformed) human lymphocytes and subjected it to reverse transcription, PCR amplification using primers located in exons 1 and 8 and sequencing. This analysis identified a reproducible, complex pattern of alternative splicing around intron 4 (similar analysis of exons 8-11 showed no evidence for alternative splicing; data not shown). We identified two main products of comparable abundance in both the total (Fig. 1c) and the poly $(\mathrm{A})^{+}$RNA fractions (data not shown), derived either from the fully spliced form or from an unspliced form retaining intron 4 (Fig. 1c). Sequence analysis of the introns and their flanking regions identified a suboptimal 5' splice site (Fig. 1b) that could reduce the efficiency of splicing, leading to the retention of intron 4 (refs. 5,6). Examples of intron retention have been found in transcripts from several species ${ }^{7}$. SNP rs175174 is located in the middle of intron 4, embedded in a highly conserved heptanucleotide motif (Fig. 1d). The retained intron introduces a stop codon in the open reading frame that could lead to premature termination of translation (Fig. 1d).

We constructed minigenes from the 'risk' and 'nonrisk' alleles containing exon 4 , intron 4 and exon 5 along with flanking genomic sequences and transfected them into human embryonic kidney (HEK293) cells. Initial semiquantitative PCR amplification analysis showed that the pattern of splicing observed in lymphocytes was recapitulated in total RNA prepared from HEK293 cells transiently transfected with the minigene (Fig. 2a). The relative amount of the splice form containing intron 4 was significantly greater in RNA from the risk minigenes (Fig. 2a). Real-time quantitative RT-PCR analysis using a different set of primers confirmed this finding and allowed an accurate estimation of the observed increase at $\sim 33 \%$ (Fig. 2b). Real-time quantitative RT-PCR on total fetal brain RNA showed that the ratio of unspliced to spliced transcript was $\sim 25 \%$ higher in samples homozygous with respect to the risk allele than in samples homozygous with respect to the nonrisk allele ( $P=0.04$; Fig. 2 c). The results were not as conclusive as our in vitro studies owing to relatively large interindividual variation and small sample sizes. Taken together with our in vitro experiments, however, these results indicate that the relative abundance of the unspliced form (and, by inference, the level of ZDHHC8 activity) is affected by the intronic variant rs 175174 .

ZDHHC8 is predicted to have four transmembrane domains and a cysteine-rich domain that includes an Asp-His-His-Cys motif and a Cys4 zinc-finger-like metal binding $\operatorname{site}^{8}$ (Fig. 3a). Two yeast ZDHHC proteins, ERF2 and AKR1, are transmembrane palmitoyltransferases $^{9,10}$, and other members of the family, including ZDHHC8, are probably also palmitoyltransferases with unidentified substrates. Immunostaining for ZDHHC8 in HeLa cells occurs throughout the cytoplasm in vesicle-like clusters but is also conspicuously concentrated

${ }^{1}$ Columbia University, College of Physicians \& Surgeons, Department of Physiology and Cellular Biophysics and Center for Neurobiology \& Behavior, New York, New York 10032, USA. ${ }^{2}$ The Rockefeller University, Human Neurogenetics Laboratory, New York, New York 10021, USA. ${ }^{3}$ These authors contributed equally to this work. Correspondence should be addressed to M.K. (karayim@rockefeller.edu) or J.A.G. (jag90@columbia.edu). 
at a perinuclear compartment (Fig. 3b). In primary developing hippocampal neurons transfected with ZDHHC8, we localized immunoreactivity to a perinuclear domain (data not shown), as well as to vesicle-like clusters along the dendritic shafts (Fig. 3c).

Palmitate reversibly modifies numerous classes of neuronal proteins, including proteins important for neuronal development, neurotransmitter receptors and synaptic scaffolding proteins ${ }^{11}$. ZDHHC8 is widely expressed in the adult human brain ${ }^{4}$. More detailed analysis of expression in the adult mouse brain showed higher expression levels in the cortex and hippocampus (Fig. 4a), areas presumed to be important in the pathophysiology of schizophrenia. We used homologous recombination to produce a knockout mouse model of ZDHHC8 deficiency (Fig. 4b). Brain morphology seemed normal in homozygous $Z d h h c 8$ mutant mice by gross evaluation and histological examination of sections (Fig. 4c). We examined the effect of ZDHHC8 deficiency in the state of sensorimotor gating, an endophenotype of schizophrenia and other neuropsychiatric diseases, which is widely studied in rodents ${ }^{12,13}$. Homozygous mutant female mice (but not male; data not shown) had significantly lower levels of prepulse inhibition than wild-type littermate controls at 78 $\mathrm{db}(P=0.01$; Fig. 4d $)$. This decrease was relatively modest, and heterozygous mice did not differ from wild-type littermate controls. Further analysis of female mice uncovered additional strong deficits in locomotor activity in an open field test of spontaneous exploratory behavior under mildly stressful conditions, such as new environment and light. Specifically, both homozygous and heterozygous $Z d h h c 8$ mutant female mice traveled significantly less distance
$(P=0.0001$ and 0.004 , respectively; Fig. 5a) and had significantly fewer rearing responses $(P=0.001$ and 0.005 , respectively; Fig. 5b) than their wild-type littermates in the open field assay. Moreover, both homozygous and heterozygous $Z d h h c 8$ mutant female mice entered the aversive centerfield significantly less frequently than their wild-type littermates $(P=0.0005$ and 0.002 , respectively; Fig. 5c) and moved significantly less in the center ( $\sim 2$ times less) but not in the margin of the field ( $\sim 1.1$ times less). These results indicate that a greater fear of new environments probably accounts for the decreased locomotor activity. The effect of ZDHHC8 deficiency in exploratory behavior seems to be sexually dimorphic, as homozygous $Z d h h c 8$ mutant male mice had only a modest deficit in locomotor activity in the open field $(P=0.03)$ and heterozygotes did not differ from wild-type littermate controls in this respect (Fig. 5d).

Given the effects of ZDHHC8 deficit on behavior, it may be relevant that protein palmitoylation modulates several neurotransmitter systems ${ }^{11}$, including activity-dependent plasticity at glutamatergic synapses ${ }^{14}$. We investigated the effects of the psychomimetic dizocilpine (MK801), which is traditionally considered to be an NMDA-receptor blocker but probably also results in secondary activation of non-NMDA receptor glutamatergic neurotransmission by increasing glutamate efflux ${ }^{15}$, in homozygous $Z d h h c 8$ mutant mice and wild-type littermate controls. We used the ratio of activity during the half-hour after the injection to the activity during the half-hour before the injection as an index of the locomotor-activating effects of the drug. In terms of ratio index, homozygous $Z d h h c 8$ mutant female (but not male) mice seem to be somewhat less sensitive to MK801-induced a

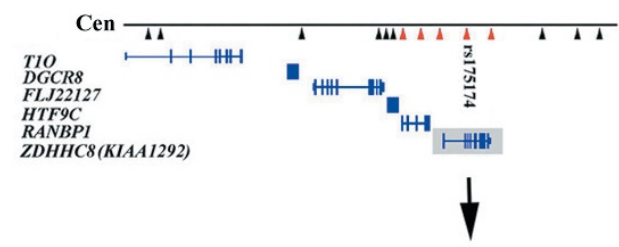

b

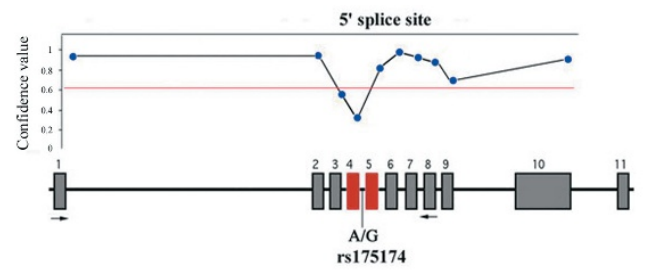

d

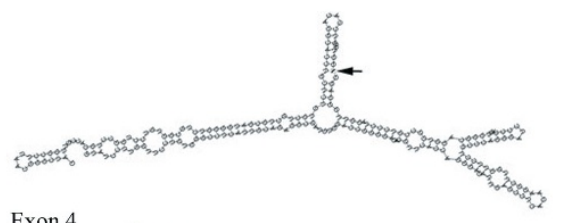

Exon 4

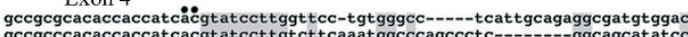
gccgcccacaccaccatcacgtatccttgtcttcaaatggcccagcctc---------ggcagcatatc
gcggcccacaccaccatcacgtatcct tgtctccacgtggccc-----tc-------gggagcacatc cggggacacgttcaccagggagattg-agcctcagaaggcttggttcctgeccagggatccccagtgggc

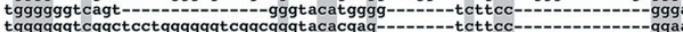
tggggggtcggctcctggggggtcggeggg aggtggccgtccctaggttgggaggnggtttgtccacaggcacctgctctgtgctgctgcgatg--ggg gtgtcgctgtgcttagg-cgagaggggg-----ccacaagaactgtcactgtagtggggggagggaggg Exon 5
ctgcggtgtcctggcctgcaccgttggccttaacgggetagcatggctgtatgtgtgt Human $\begin{array}{ll}\text { cttc-atgttcttgcctcaactgttggccttaacaggcagcatggctgtcatgtgtgt } & \text { Mouse } \\ \text { cgtc-gtgttcttgcctcaactgttggcttaacaggccagcatggctgtcatgtgtgt } & \text { Rat }\end{array}$

C
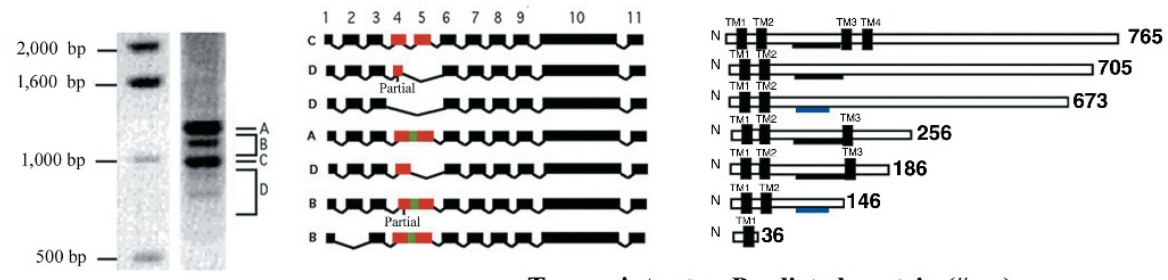

Transcript $\rightarrow$ Predicted protein (\# aa)

Figure 1 Genomic analysis of the ZDHHC8 locus. (a) Associated subregion at the distal part of the 22q11 locus. Arrowheads indicate the SNPs tested in the 80-kb haplotypic block ${ }^{4}$. Red arrowheads indicate the five SNPs associated with schizophrenia in the families we tested ${ }^{4}$. The position of the most significantly associated SNP, rs 175174, in intron 4 of ZDHHC8 is indicated. Blue bars represent the approximate distribution of exons of genes residing in or immediately adjacent to the 80-kb haplotypic block. (b) Genomic organization of ZDHHC8. The exon-intron structure of ZDHHC8 is shown, along with the position of SNP rs175174 in intron 4. Genomic sequence of ZDHHC8 was drawn from the April 2002 freeze of the University of California Santa Cruz database. Confidence values for the $5^{\prime}$ splice sites were calculated by using NetGene2 worldwide web server. (c) Identification of alternatively spliced forms of ZDHHC8 and analysis of the predicted proteins. The predicted protein sequences were analyzed using TMHMM prediction server to predict transmembrane helices and CD-Search to identify the ZDHHC domain. (d) Sequence and predicted secondary structure of ZDHHC8 intron 4. A sequence alignment of ZDHHC8 intron 4 from human, mouse and rat is shown, indicating blocks of conserved sequence identity (shaded). The suboptimal 5' splice site is indicated by two dots; the position of rs175174 is indicated by an arrowhead; the position of the in-frame premature stop codon is indicated by asterisks. 

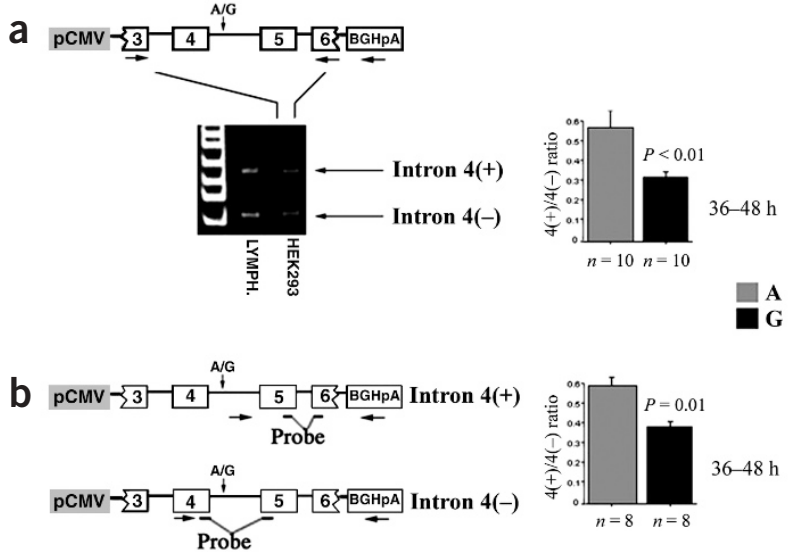

C
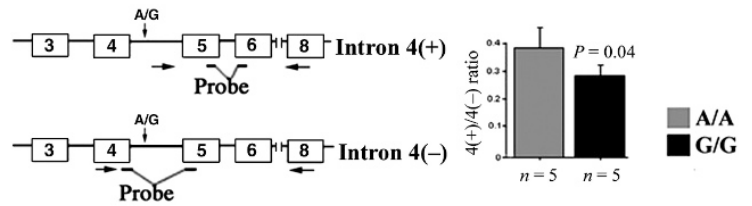

stimulation of locomotor activity than wild-type littermate controls $(P=0.05$; Fig. 5e,f). This result suggests that $Z D H H C 8$ affects behavior at least partly by interfering with glutamatergic transmission. In that respect, it is worth noting that the behavioral effects of MK801 seem to be sexually dimorphic in pharmacological animal models of schizophrenia symptoms also ${ }^{16}$. Further analysis will be needed to dissect the neural pathways affected by ZDHHC8 deficiency.

We examined whether a similar sexual dimorphism exists in functional variants of $\mathrm{ZDHHC8}$ and schizophrenia in humans. We

Figure 3 Structure and subcellular localization of ZDHHC8 gene product. (a) The ZDHHC family of putative palmitoyltransferases. Homology searches found at least ten ZDHHC members in the human genome. The predicted ZDHHC8 domain structure is shown for the transmembrane domains (I-IV) and the ZDHHC domain (blue). Also shown is a sequence alignment of ZDHHC motifs from humans, indicating essential residues of the ZDHHC domain (underlined), including a DHHC motif consisting of the amino acids Asp-HisHis-Cys and a cysteine-rich domain that includes a Cys4 zinc-finger-like metal binding site. (b) Subcellular localization of ZDHHC8 (red signal). Expression of the cis-Golgi marker GM130, trans-Golgi marker golgin-97, late endosomal marker mannose 6-phosphate receptor (M6PR) and early endosomal marker EEA1 (green signal). We did not find any overlap between ZDHHC8, the endoplasmic reticulum markers Grp78 and calnexin (data not shown) and the nuclear marker To-Pro-3 iodide. The nucleus is indicated by the blue signal. Identical localization patterns were obtained with two separate ZDHHC8 forms with presumably impaired palmitoyltransferase activity: a form with a C134A mutation introduced into the DHHC motif (DHHA) of the ZDHHC domain and an alternative splicing form, which lacks a DHHC-CRD domain. This suggests that the subcellular distribution pattern is independent of the enzymatic activity (data not shown). (c) The distribution of ZDHHC8 in cultured hippocampal neurons. Neurons (DIV9) cotransfected with ZDHHC8 and yellow fluorescent protein (YFP)-tagged postsynaptic density-95 (PSD-95) were stained with 280GP antibody (red signal). YFP-PSD-95 expression was visualized at low magnification (i) and high magnification (iii) by direct fluorescence (green signal). Endogenous GRIN2B was stained with GRIN2B antibody (ii) (blue signal). ZDHHC8 immunoreactivity was localized to vesicle-like clusters along the dendritic shafts. Occasionally, ZDHHC8 immunoreactivity colocalized along the processes with PSD-95 clusters at the developing dendritic spines. No staining was observed in control sections when primary antibodies were omitted (data not shown).
Figure 2 SNP rs 175174 modulates the levels of the ZDHHC8 form that retains intron 4. (a) Semiquantitative polyacrylamide gel electrophoresis of the minigene products. Total RNA was isolated from HEK293 cells transfected with the ZDHHC8 minigene and was reverse-transcribed using primer R-BGHpA to distinguish the minigene from the endogenous $Z D H H C 8$ gene. The cDNA was then amplified using the primer pair F2-ZDHHC8 and R2-ZDHHC8 from exons 3 and 6 , respectively. The bar graph shows the ratio of the unspliced to the fully spliced form. (b,c) Quantification of retention of intron 4 of ZDHHC8 using real-time quantitative PCR assay. Bar graphs show the ratio of the unspliced to the fully spliced form. The primer pair F4ZDHHC8 and R-BGHpA and Probe1-ZDHHC8 were used to quantify amplicon intron 4(-); the primer pair F5-ZDHHC8 and R-BGHpA and Probe2-ZDHHC8 were used to quantify amplicon intron $4(+)$. The relative level of the expression was calculated from the ratio of the intron $4(+)$ cycle threshold (Ct) to intron $4(-)$ Ct values, both of which were normalized to the control transcript GAPDH Ct value using the formula $2 \times(\Delta \mathrm{Ct}$ (intron $4(+)-$ $\Delta \mathrm{Ct}$ (intron $4(-))$, in which $\Delta \mathrm{Ct}$ represents the difference in $\mathrm{Ct}$ values between the minigene transcript and the control transcript. A similar protocol was used for real-time quantitative RT-PCR on total fetal brain RNA (c), except that a primer from exon 8 was used. The genotype at SNP rs 175174 is indicated; $A$ is the risk allele and $G$ is the nonrisk allele.

examined allele transmission of SNP rs175174 by gender in an extended sample of 389 families from the US and South Africa that included the families described in our original study. In this extended sample we confirmed the presence of a sexually dimorphic

a

a
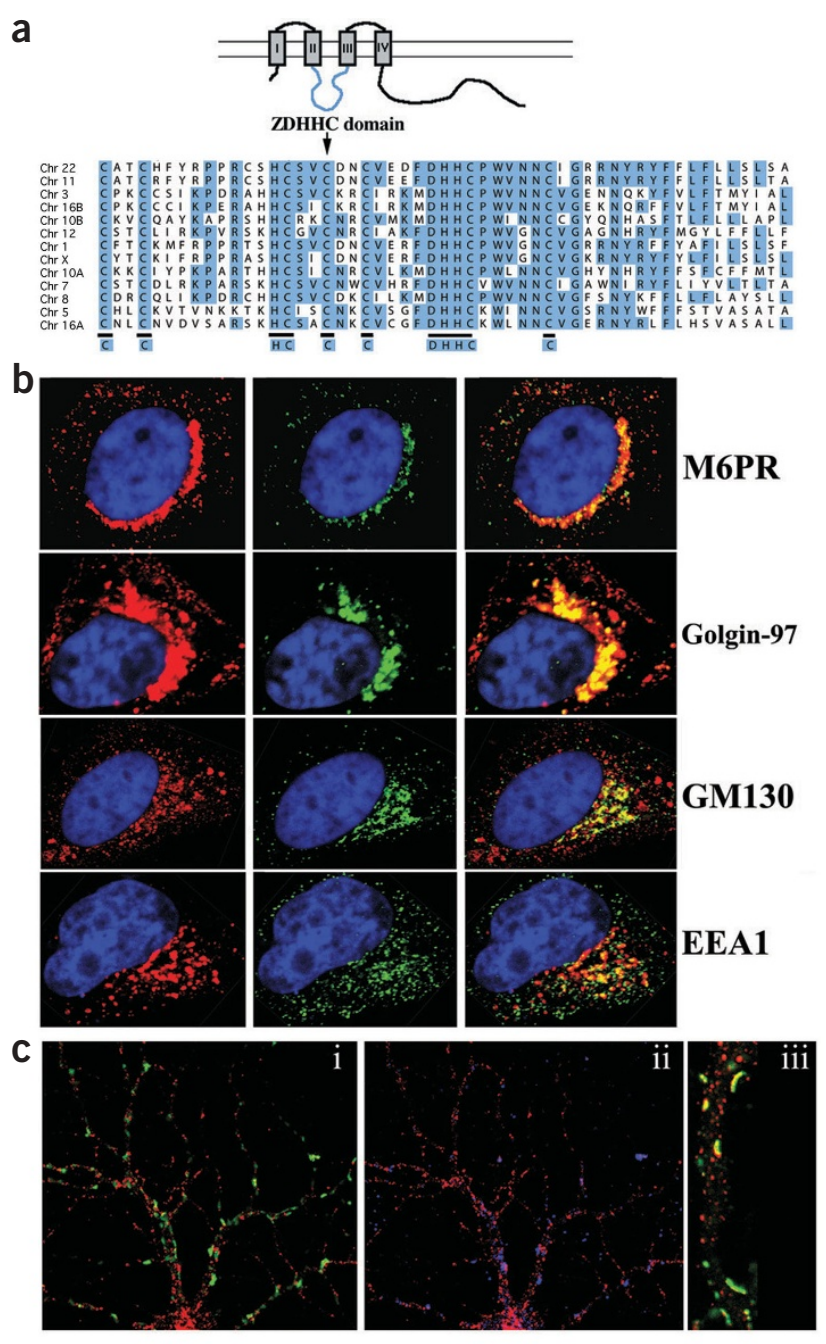
effect that we initially observed, to a lesser extent, in our discovery sample $^{4}$ (Table 1). Specifically, we identified a sex-related heterogeneity of allele transmission (heterogeneity $\chi^{2}=8.2, P=0.004$ ). The effect of SNP rs 175174 was significant in females $\left(\chi^{2}=12.2, P=\right.$ 0.0005 , transmitted:untransmitted ratio $=82: 43)$ but not in males $\left(\chi^{2}=0.02, P=0.88\right.$, transmitted:untransmitted ratio $\left.=106: 108\right)$.

The interspecies consistency in sex-specific phenotypic differences associated with low ZDHHC8 levels, taken together with the functional effect of the associated variant, the expression profile, neuronal localization, putative function and haploinsufficiency of $Z D H H C 8$, supports the idea that $Z D H H C 8$ is a good candidate for a gene contributing to the association of the 22q11 locus with susceptibility to schizophrenia.

a

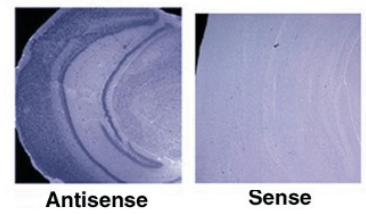

b

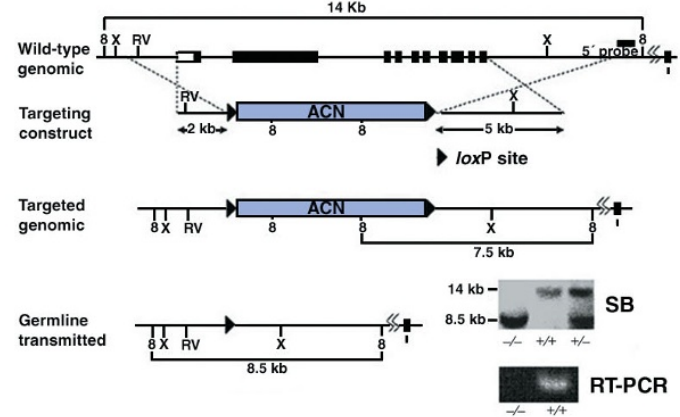

C

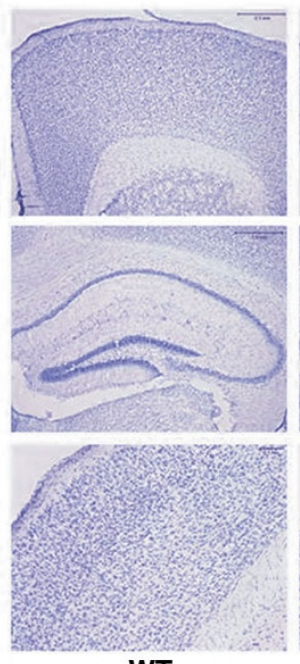

WT

d

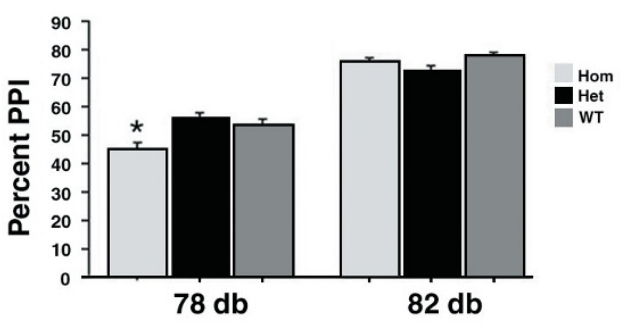

In concluding on the basis of unspliced:spliced ratios that the effect of rs 175174 is modest, several points should be noted. First, the truncated protein encoded by the unspliced form, if translated, may act in a dominant negative way, leading to further decrease in activity. Second, although the unspliced product bearing a premature termination codon efficiently escapes nonsense-mediated decay in fresh lymphocytes and HEK293 cells (Figs. 1 and 2; J.M. and J.A.G., unpublished data), the extent to which this is true in other cell types is unknown ${ }^{17}$. Third, the present study shows that SNP rs175174 is one of the causative variants but does not rule out the existence of additional interacting functional variants on the risk haplotype $e^{4,18,19}$. Finally, our analysis shows that even a modest reduction of ZDHHC8 levels can have substantial effects on behavior and, consequently, on disease pathophysiology.

The sexually dimorphic effect of ZDHHC8 in schizophrenia is puzzling, but not unprecedented, and may be related to observed sex differences in onset, incidence and severity course of schizophrenia ${ }^{20-22}$. Two studies involving large samples of schizophrenic individuals also identified female-specific effects for variants from two genes that are good candidates for association with susceptibility to schizophrenia $^{23,24}$, including the gene COMT, also located on 22q11.

Systematic approaches and candidate gene studies designed to identify genes in the 22q11 region associated with susceptibility to schizophrenia have implicated the genes $P R O D H^{3,25,26}, C O M T^{20,27}$ and ZDHHC8 (ref. 4 and this study) so far. Studies of animal models suggest that deficiency of the genes on 22q11 associated with susceptibility to schizophrenia seems to modulate disease risk both by impairing synaptic function and by failing to compensate for such impairment (M. Paterlini, M.K. and J.A.G., unpublished data). Further behavioral studies modeling additional schizophrenia endophenotypes, as well as neurochemical and gene profiling analysis of Zdhhc8-deficient mice, will address whether and how deficiency of ZDHHC8 fits into this emerging picture.

\section{METHODS}

Identification of alternatively spliced forms of $Z D H H C 8$. We purified total RNA from fresh human lymphocytes and reverse transcribed it using a random hexamer. We then amplified the transcript of ZDHHC8 by using primer pair F1-ZDHHC8 and R1-ZDHHC8 from exons 1 and 8, respectively, separated the PCR products on a $1.2 \%$ agarose gel, purified them and sequenced them. We

Figure 4 Targeted inactivation of the mouse Zdhhc 8 locus. (a) Brain expression of Zdhhc8. In situ hybridization analysis on adult mouse brain using a probe from the 3' untranslated region of Zdhhc8. (b) Targeting of the mouse Zdhhc8 locus in embryonic stem cells and mice. To construct the targeting construct, a Zdhhc8 genomic fragment encompassing exons 2-11 was replaced by the self-excisable pACN cassette including the neo selectable marker ${ }^{29}$. The probe used for Southern-blot (SB) analysis is shown as a thick black line. The diagnostic $\mathrm{BamH1}$ restriction fragments $(7.5$ or $8.5 \mathrm{~kb}$ ) are underlined. Results of genomic Southern-blot analysis of DNA from tail biopsy samples and brain RT-PCR are shown. Wild-type and recombinant $\mathrm{BamH} 1$ restriction fragments are $14 \mathrm{~kb}$ and $8.5 \mathrm{~kb}$, respectively. (c) Gross brain morphology. Representative photomicrographs showing Nissl staining from adult female wild-type (WT; left) and homozygous Zdhhc8 mutant mice (Hom; right) in frontal cortex ( $1.94 \mathrm{~mm}$ from Bregma; top), dorsal hippocampus (approximately $-1.46 \mathrm{~mm}$ from Bregma; middle) and auditory cortex (-2.92 $\mathrm{mm}$ from Bregma; bottom). (d) Sensorimotor gating. Prepulse inhibition assay using combinations of one startle level $(120 \mathrm{~dB})$ and two prepulse levels (78 dB and $82 \mathrm{~dB}$ ) in homozygous (Hom) and heterozygous (Het) Zdhhc8 mutant female mice and wild-type (WT) littermate controls. Higher $y$-axis values represent greater percent prepulse inhibition. No significant decreases in startle response amplitudes were identified among genotypes (data not shown). Statistical analysis was done using ANOVA repeated measures. ${ }^{*} P<0.05$ versus wild-type control. 

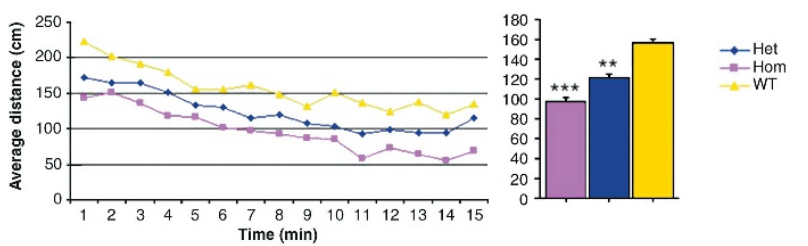

C
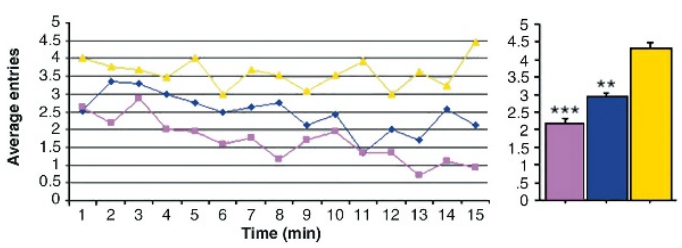

e

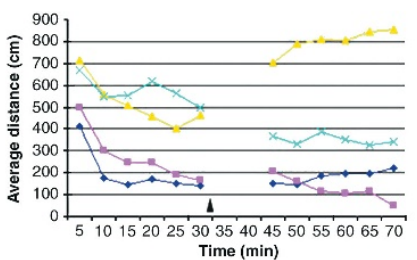

b

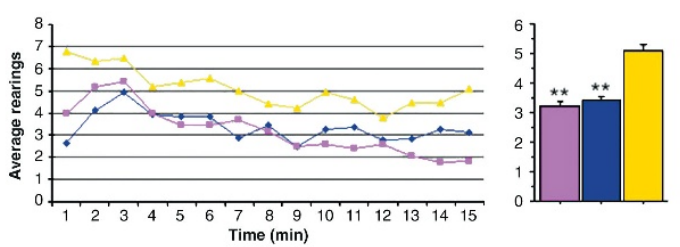

d
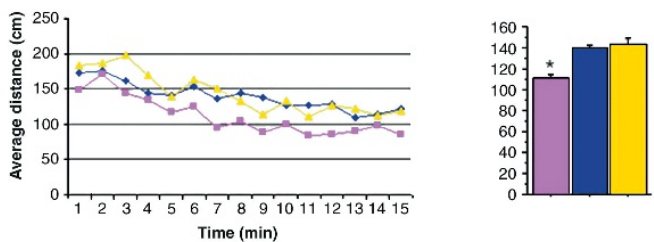

f

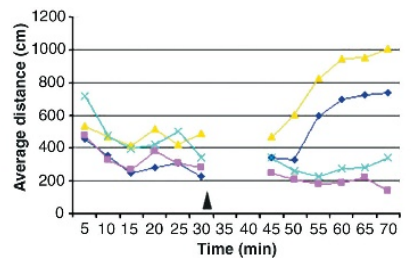

Figure 5 Targeting of the mouse Zdhhc8 locus affects exploratory activity. (a-d) Locomotor response to novelty. (a-c) Three measures of exploratory activity in response to novelty are shown in homozygous and heterozygous Zdhhc8 mutant female mice and wild-type littermate control mice: (a) total distance traveled, (b) number of rearings (vertical moves) and (c) number of entries into the centerfield. (d) Total distance traveled is shown for homozygous and heterozygous Zdhhc8 mutant male mice and wild-type littermate control mice. Both total counts (bar graphs) and time course of the effect of the mutation over 5-min intervals (line graphs) are shown for each measurement. Het, heterozygous; Hom, homozygous; WT, wild-type. (e,f) Locomotor activating effects of MK801. Locomotor stimulation after MK801 injection was observed in both genotypes in both females (e) and males (f). Homozygous Zdhhc 8 mutant females were less sensitive to MK801 in terms of the ratio of activity before injection to activity after injection. Time course of the locomotor activity for 30 min before and 30 min after injection of MK801 or vehicle is shown over 5-min intervals for each genotype. Bar graphs show the pre- to post-injection total activity ratios. Arrowheads indicate the time of MK801 injection. (a-f) ${ }^{*} P<0.05,{ }^{*} P<0.01$ and ${ }^{* * *} P<0.001$ versus wild-type controls.

subcloned fragment $\mathrm{B}$, which gave mixed signals in sequencing, and purified and sequenced plasmids from 20 isolated single colonies. We edited and translated to amino acids the DNA sequences of the alternatively spliced forms of ZDHHC 8 by using software Editseq (DNA Star). Primer sequences are available on request.

Construction and analysis of $Z D H H C 8$ minigenes. We used the primer pair F6-ZDHHC8 and R6-ZDHHC8 to amplify the genomic sequence surrounding the alternatively spliced exons 4 and 5 of ZDHHC8. We used human genomic DNA homozygous with respect to risk or nonrisk alleles at SNP rs175174 as the PCR template. We tailed the forward primer with EcoRV and the reverse primer with NotI to facilitate cloning. We purified the PCR product using Qiaquick PCR Purification Kit (Qiagen), digested it with EcoRV and NotI (New England Biolabs), purified it using Qiaquick Gel Purification Kit and ligated it into vector pcDNA3.1 (Invitrogen), which was predigested with EcoRV and NotI. We then transformed the ligation product into DH5 $\alpha$. We isolated plasmids from the transformants and sequenced them to confirm the ZDHHC8 genomic sequence and the genotypes for SNP rs175174. No variation apart from the rs175174 $\mathrm{A} \rightarrow \mathrm{G}$ was present. We purified plasmids of ZDHHC8 minigene constructs using Endofree Plasmid Maxi Kit (Qiagen) and transfected them into HEK293 cells using Lipofectamine 2000 transfection reagent (Life Technologies). We maintained HEK293 cells in Dulbecco's modified Eagle's medium (DMEM) supplemented with $10 \%$ fetal calf serum, $100 \mathrm{U} \mathrm{ml}^{-1}$ of penicillin, $100 \mu \mathrm{g} \mathrm{m}{ }^{-1}$ of streptomycin and $2 \mathrm{mM} \mathrm{L-glutamine}$ at $37^{\circ} \mathrm{C}$ under a humidified atmosphere containing $5 \% \mathrm{CO}_{2}$. One day before the transfection, we plated $5 \times 10^{5}$ cells on a $35-\mathrm{mm} \times 10$ - $\mathrm{mm}$ cell culture dish (Corning) in the growth medium without antibiotics. On the day of the transfection, we combined $4 \mu \mathrm{g}$ of DNA diluted in $250 \mu \mathrm{l}$ of DMEM with 10 $\mu \mathrm{l}$ of LipofectAmine-2000 (Invitrogen) diluted in $250 \mu \mathrm{l}$ of DMEM. We then incubated the mixture at room temperature for $20 \mathrm{~min}$ and added it to the cell culture dish. We further incubated the cells at $37^{\circ} \mathrm{C}, 5 \% \mathrm{CO}_{2}$ for $36-48 \mathrm{~h}$. We purified total RNA from the transfected cells using RNeasy Mini Purification kit according to the manufacturer's instructions (Qiagen). Primer sequences are available on request.

Quantification of ZDHHC8 minigene expression. For semiquantitative polyacrylamide gel electrophoresis, we carried out PCR using Herculase Enhanced DNA Polymerase following the manufacturer's instructions (Stratagene), except that we used $\left[\alpha{ }^{3}{ }^{32} \mathrm{P}\right] \mathrm{dCTP}$ instead of dCTP to label the PCR product. We used a touchdown protocol for the amplifications, consisting of denaturation at $94^{\circ} \mathrm{C}$ for $3 \mathrm{~min} ; 15$ cycles of $94^{\circ} \mathrm{C}$ for $30 \mathrm{~s}, 68^{\circ} \mathrm{C}$ (minus $1{ }^{\circ} \mathrm{C}$ per cycle) for $45 \mathrm{~s}$ and $72{ }^{\circ} \mathrm{C}$ for $45 \mathrm{~s} ; 5$ cycles of $94^{\circ} \mathrm{C}$ for $30 \mathrm{~s}, 53^{\circ} \mathrm{C}$ for $45 \mathrm{~s}$ and $72^{\circ} \mathrm{C}$ for $45 \mathrm{~s}$; and a final extension cycle of $72^{\circ} \mathrm{C}$ for $7 \mathrm{~min}$. We separated the PCR products on $4-12 \%$ polyacrylamide gels (Invitrogen), exposed the gels to KODAK X-OMAT AR film and quantified the band densities by using MCID 4.0 (Imaging Research). For real-time quantitative (Taqman) PCR

\section{Table 1 Transmission of the risk allele rs 175174-A}

\begin{tabular}{lcccl}
$\begin{array}{l}\text { Sample } \\
\text { (number of } \\
\text { families) }\end{array}$ & Sex & Transmitted & Untransmitted & $P$ value \\
\hline Discovery (106) & F & 30 & 10 & 0.0016 \\
Total (389) & M & 35 & 22 & 0.08 \\
& F & 82 & 43 & 0.0005 \\
& M & 106 & 108 & 0.88 \\
\hline
\end{tabular}


assay, we transcribed the total RNA using Taqman Reverse Transcription Reagents (Applied Biosystems). We used a random hexamer as the primer for reverse transcription. We amplified the transcripts from the minigene by using Taqman Universal PCR Master Mix following the manufacturer's instructions (Applied Biosystems). We carried out quantitative PCR in an ABI Prism 7700 Sequence Detection System (Applied Biosystems).

Cell culture and transfection. We obtained primary cultures of hippocampal neurons from mouse embryos at embryonic day 15-16. We isolated the entire hippocampus, dissociated it with trypsin treatment and trituration and plated cells on poly-L-lysine-coated 12-mm glass coverslips at a cell density of $4 \times 10^{4}$. We maintained neurons in Neurobasal medium (Gibco) supplemented with B 27 (Gibco), $0.5 \mathrm{mM}$ L-glutamine, penicillin and streptomycin. For transfection of ZDHHC8 and YFP-PSD-95, we transfected hippocampal cultures with $0.5 \mu \mathrm{g}$ of DNA using LipofectAmine-2000 in Neurobasal medium supplemented with B-27 and $0.5 \mathrm{mM}$ L-glutamine. We obtained HeLa cells from the American Type Culture Collection and maintained them in DMEM supplemented with $10 \%$ fetal bovine serum. We transiently transfected HeLa cells $(2 \times$ $10^{4}$ per 12-mm coverslip) with $0.5 \mu \mathrm{g}$ of DNA using LipofectAmine-2000 in DMEM supplemented with $10 \%$ fetal bovine serum and cultured them for $18 \mathrm{~h}$. We constructed the expression vector for human ZDHHC8 by PCR amplification of $Z \mathrm{DHHC}$ coding sequence, digestion with EcoRI and $B a m \mathrm{HI}$ and ligation of the resulting fragment into EcoRI-BamHI-digested pcDNA3.1/Myc-His(-)C. Primer sequences are available on request.

Immunocytochemistry. We obtained the 280GP antibody by immunizing guinea pigs with a synthetic peptide derived from the C-terminal sequence of human ZDHHC8 (KKVSGVGGTTYEISV). Pilot experiments indicated that 280GP recognizes only exogenously transfected protein with high specificity (data not shown). For double-staining of ZDHHC8 and NMDA receptor, we fixed neurons with methanol for $10 \mathrm{~min}$ at $-20^{\circ} \mathrm{C}$, blocked them with $1 \%$ bovine serum albumin and $1 \%$ normal goat serum and incubated them with $280 \mathrm{GP}$ and mouse monoclonal antibody to GRIN2B (NMDAR2B; BD Transduction Laboratories) at 1:200 dilutions for $1 \mathrm{~h}$ at room temperature. After rinsing them several times with phosphate-buffered saline, we incubated cells with the antibody to guinea pig IgG conjugated to Cy3 (Jackson ImmunoResearch Laboratory; diluted 1:100 in blocking solution) and antibody to mouse IgG conjugated to Cy5 (Jackson ImmunoResearch Laboratory; diluted 1:100 in blocking solution) at room temperature for $30 \mathrm{~min}$. We fixed HeLa cells with $3.7 \%$ paraformaldehyde for $30 \mathrm{~min}$ at room temperature and permeabilized them with $0.1 \%$ sodium citrate containing $0.1 \%$ Triton X-100 for 20 min on ice. After blocking with $1 \%$ bovine serum albumin and $1 \%$ normal goat serum for $1 \mathrm{~h}$ at room temperature, we incubated cells with mouse monoclonal antibodies to EEA1 (BD Transduction Laboratories) diluted 1:50, to mannose 6-phosphate receptor (Affinity Bioreagents) diluted 1:100, to GM130 (BD Transduction Laboratories) diluted 1:100 and to golgin-97 (Molecular Probes) diluted 1:100 as primary antibodies. After several rinses with phosphate-buffered saline, we incubated cells with the antibody to guinea pig IgG conjugated to Cy3 and antibody to mouse IgG conjugated to Alexa Fluor 488 (Molecular Probes; diluted 1:100 in blocking solution) at room temperature for $30 \mathrm{~min}$. We used To-Pro-3 iodide (Molecular Probes) to visualize the nucleus. We mounted coverslips on Vectashield (Vector Laboratory). We captured and analyzed fluorescent images of cells on an LSM510 Meta Confocal laser-scanning microscope (Zeiss).

Generation of Zdhhcs knockout mice. We carried out homologous recombination in embryonic stem cells and generated chimeric mice essentially as previously described ${ }^{28}$. Approximately $5 \%$ of the tested embryonic stem cell clones were positive for homologous recombination, and two clones were selected for injection into C57B6 blastocysts. Chimeric males were mated with C57BL/6 females, and we genotyped DNA from tail biopsy samples from $\mathrm{F}_{1}$ agouti-coat pups by Southern blotting and PCR at the Zdhhc8 genomic locus. We mated $\mathrm{F}_{1}$ heterozygous mice and obtained $\mathrm{F}_{2}$ mice of all three genotypes. Zdhhc8 homozygous mutant mice were viable, fertile and had normal nesting behavior. The mutation was on a hybrid C57BL/ $6 \times 129$ Sv background. All animal procedures were done according to protocols approved by the Institutional Animal Care and Use Committees established by Rockefeller and Columbia Universities under the federal and state regulations.
Gross brain morphology. We analyzed brain morphology in four homozygous $Z d h h c 8$ mutant and four wild-type littermate mice, 9-15 weeks of age. We postfixed brains in $4 \%$ paraformaldehyde and transferred them to $30 \%$ sucrose at $4{ }^{\circ} \mathrm{C}$ until they sank. Using a sliding microtome, we cut $40-\mu \mathrm{m}$ coronal sections. We stained one series of every four sections through the entire brain with Nissl and cresyl violet (Sigma). Regions of interest were digitally photographed (Spot RT Camera, Diagnostic Instruments) at 40× and 100× magnification (Nikon E800 microscope). We analyzed the photographs using NIH Image program (1.63).

Assay for prepulse inhibition of the acoustic startle response. We housed adult (4-month-old) homozygous $(n=19)$ and heterozygous $(n=23) Z d h h c 8$ mutant mice and wild-type littermate controls $(n=21)$ individually for 2 weeks before testing prepulse inhibition in a SR-Lab system (San Diego Instruments). We calculated response amplitude as the maximum response level occurring during the 100-ms recording. Because mice can, in principle, habituate to the prepulse, as well as to the startle stimulus, we kept the number of trials to the essential minimum. We placed each mouse in the chamber and gave it a 5-min acclimation period, during which background noise (67 dB) was continually present. Each mouse then received eight sets of four trial types distributed pseudorandomly and separated by an average 15 -s intertrial interval: trial 1, 40-ms, 120-dB noise burst alone; trials 2 and 3, 120-dB startle stimulus preceded $100 \mathrm{~ms}$ earlier by a $20-\mathrm{ms}, 78-\mathrm{dB}$ or $82-\mathrm{dB}$ noise burst (prepulse); trial 4, no stimulus, background noise alone (67 $\mathrm{dB})$. We analyzed data using ANOVA repeated measures.

Open field assay. Mice were not exposed to the chamber before testing. We monitored activity in a clear acrylic chamber directly illuminated and equipped with infrared sensors to automatically record horizontal and vertical activity (Colbourn Instruments). We placed each mouse initially in the periphery of the chamber and recorded the total distance traveled, the number of rearings (vertical moves) and the number of entries in the centerfield over the next $15 \mathrm{~min}$ as a measure of a locomotor response to novelty. We tested 47 female mice (17 homozygous $Z d h h c 8$ mutant, 17 heterozygous $Z d h h c 8$ mutant and 13 wildtype littermate controls) and 45 male mice (13 homozygous Zdhhc8 mutant, 19 heterozygous $Z d h h c 8$ mutant and 13 wild-type littermate controls).

Drug administration. We purchased MK801 from Sigma. Each experimental group consisted of 8-10 mice, which received the same number of injections, administered intraperitoneally, of either drug ( $0.4 \mathrm{mg}$ per $\mathrm{kg}$ body weight) or vehicle. After $30 \mathrm{~min}$ of prehabituation in the open field, we removed the mice, injected them and left them to rest for $10 \mathrm{~min}$ in their home cage before returning them to the open field arena, where we monitored them for an additional $30 \mathrm{~min}$.

Fetal brain samples. We obtained tissues from the tissue collection and distribution program at the University of Washington, Laboratory for the study of embryology. The tissues were from 90-120-d-old specimens and were snapfrozen in liquid nitrogen.

Samples from affected families. The US and South African families with schizophrenia were described in detail elsewhere ${ }^{3,4}$. All methods were approved by Institutional Review Boards at participating sites and all participants signed appropriate informed consent. We calculated allele transmission using the TDT method as described elsewhere ${ }^{3,4}$.

URLs. The University of California Santa Cruz database is available at http:// genome.ucsc.edu/. The NetGene2 worldwide web server and the TMHMM prediction server are available at http://www.cbs.dtu.dk. CD-Search is available at http://www.ncbi.nlm.nih.gov/Structure/cdd/cdd.shtml.

\section{ACKNOWLEDGMENTS}

We thank J. Keegan, C. Frazier and M. Monsey for technical support; P. Scheiffele for advice; and Y.-J. Chen for help in the initial phases of this project. This study was supported by grants from the US National Institute of Mental Health (to M.K. and J.A.G.) and the New York Academy of Sciences (to J.A.G.). J.A.G. is also the recipient of a McKnight Brain Disorders Award and a Vicente NARSAD Young Investigator. 
COMPETING INTERESTS STATEMENT

The authors declare that they have no competing financial interests.

Received 29 March; accepted 7 May 2004

Published online at http://www.nature.com/naturegenetics/

1. Karayiorgou, M. et al. Schizophrenia susceptibility associated with interstitial deletions of chromosome 22q11. Proc. Natl. Acad. Sci. USA 92, 7612-7616 (1995).

2. Bassett, A.S. et al. The schizophrenia phenotype in 22q11 deletion syndrome. Am. J. Psychiatry 160, 1580-1586 (2003).

3. Liu, H. et al. Genetic variation at the 22q11 PRODH2/DGCR6 locus presents an unusual pattern and increases susceptibility to schizophrenia. Proc. Natl. Acad. Sci. USA 99, 3717-3722 (2002).

4. Liu, H. et al. Genetic variation in the $22 \mathrm{q} 11$ locus and susceptibility to schizophrenia. Proc. Natl. Acad. Sci. USA 99, 16859-16864 (2002).

5. Dirksen, W.P., Sun, Q. \& Rottman, F.M. Multiple splicing signals control alternative intron retention of bovine growth hormone pre-mRNA. J. Biol. Chem. 270, 5346-5352 (1995).

6. Huang, Y. \& Carmichael, G.G. A suboptimal $5^{\prime}$ splice site is a cis-acting determinant of nuclear export of polyomavirus late mRNAs. Mol. Cell. Biol. 16, 6046-6054 (1996).

7. Kienzle, N. et al. Intron retention may regulate expression of Epstein-Barr virus nuclear antigen 3 family genes. J. Virol. 73, 1195-1204 (1999).

8. Linder, M.E. \& Deschenes, R.J. New insights into the mechanisms of protein palmitoylation. Biochemistry 42, 4311-4320 (2003).

9. Roth, A.F., Feng, Y., Chen, L. \& Davis, N.G. The yeast DHHC cysteine-rich domain protein Akr1p is a palmitoyl transferase. J. Cell. Biol. 159, 23-28 (2002).

10. Lobo, S., Greentree, W.K., Linder, M.E. \& Deschenes, R.J. Identification of a Ras palmitoyl-transferase in Saccharomyces cerevisiae. J. Biol. Chem. 277, 41268-41273 (2002).

11. El-Husseini, A.el-D. \& Bredt, D.S. Protein palmitoylation: a regulator of neuronal development and function. Nat. Rev. Neurosci. 3, 791-802 (2002).

12. Swerdlow, N.R. \& Geyer, M.A. Using an animal model of deficient sensorimotor gating to study the pathophysiology and new treatments of schizophrenia. Schizophr. Bull. 24, 285-301 (1998).

13. Gogos, J.A. et al. The gene encoding proline dehydrogenase modulates sensorimotor gating in mice. Nat. Genet. 21, 434-439 (1999).

14. El-Husseini, A.el-D. et al. Synaptic strength regulated by palmitate cycling on PSD-
95. Cell 108, 849-863 (2002)

15. Moghaddam, B. \& Adams, B.W. Reversal of phencyclidine effects by a group II metabotropic glutamate receptor agonist in rats. Science 281, 1349-1352 (1998).

16. D'Souza, D.N., Harlan, R.E. \& Garcia, M.M. Sexual dimorphism in the response to Nmethyl-D-aspartate receptor antagonists and morphine on behavior and c-Fos induction in the rat brain. Neuroscience 93, 1539-1547 (1999).

17. Bateman, J.F., Freddi, S., Nattrass, G. \& Savarirayan, R. Tissue-specific RNA surveillance? Nonsense-mediated mRNA decay causes collagen $X$ haploinsufficiency in Schmid metaphyseal chondrodysplasia cartilage. Hum. Mol. Genet. 12, 217-225 (2003).

18. Drysdale, C.M. et al. Complex promoter and coding region beta 2-adrenergic receptor haplotypes alter receptor expression and predict in vivo responsiveness. Proc. Natl. Acad. Sci. USA 97, 10483-10488 (2000).

19. Peltekova, V.D. et al. Functional variants of OCTN cation transporter genes are associated with Crohn disease. Nat. Genet. 36, 471-475 (2004).

20. Aleman, A., Kahn, R.S. \& Selten, J.P. Sex differences in the risk of schizophrenia evidence from meta-analysis. Arch. Gen. Psychiatry 60, 565-571 (2003).

21. Leung, A. \& Chue, P. Sex differences in schizophrenia, a review of the literature. Acta Psychiatr. Scand. Suppl. 401, 3-38 (2000).

22. Konnecke, R., Hafner, H., Maurer, K., Loffler, W. \& an der Heiden, W. Main risk factors for schizophrenia: increased familial loading and pre- and peri-natal complications antagonize the protective effect of oestrogen in women. Schiz. Res. 44, 81-93 (2000).

23. Shifman, S., et al. A highly significant association between a COMT haplotype and schizophrenia. Am. J. Hum. Genet. 71, 1296-1302 (2002).

24. Hennah, W. et al. Haplotype transmission analysis provides evidence of association for DISC1 to schizophrenia and suggests sex-dependent effects. Hum. Mol. Genet. 12, 3151-3159 (2003)

25. Jacquet, H. et al. PRODH mutations and hyperprolinemia in a subset of schizophrenic patients. Hum. Mol. Genet. 11, 2243-2249 (2002).

26. Li, T., et al. Evidence for association between novel polymorphisms in the PRODH gene and schizophrenia in a Chinese population. Am. J. Med. Genet. published online 24 May 2004. (doi:10.1002/ajmg.b.30049).

27. Egan, M.F. et al. Effect of COMT Val108/158 Met genotype on frontal lobe function and risk for schizophrenia. Proc. Natl. Acad. Sci. USA 98, 6917-6922 (2001).

28. Gogos, J.A., Osborne, J., Nemes, A., Mendelsohn, M. \& Axel, R. Genetic ablation and restoration of the olfactory topographic map. Cell 103, 609-620 (2000).

29. Bunting, M., Bernstein, K.E., Greer, J.M., Capecchi, M.R. \& Thomas, K.R. Targeting genes for self-excision in the germ line. Genes Dev. 13, 1524-1528 (1999). 